ISSN 1855-3966 (printed edn.), ISSN 1855-3974 (electronic edn.)

ARS MATHEMATICA CONTEMPORANEA 18 (2020) 339-357

https://doi.org/10.26493/1855-3974.1998.2f4

(Also available at http://amc-journal.eu)

\title{
The thickness of the Kronecker product of graphs*
}

\author{
Xia Guo \\ School of Mathematical Sciences, Xiamen University, Xiamen, P. R. China \\ Yan Yang ${ }^{\dagger}$ \\ School of Mathematics, Tianjin University, Tianjin, P. R. China
}

Received 7 May 2019, accepted 10 May 2020, published online 22 October 2020

\begin{abstract}
The thickness of a graph $G$ is the minimum number of planar subgraphs whose union is $G$. In this paper, we present sharp lower and upper bounds for the thickness of the Kronecker product $G \times H$ of two graphs $G$ and $H$. We also give the exact thickness numbers for the Kronecker product graphs $K_{n} \times K_{2}, K_{m, n} \times K_{2}$ and $K_{n, n, n} \times K_{2}$.
\end{abstract}

Keywords: Thickness, Kronecker product graph, planar decomposition.

Math. Subj. Class. (2020): 05C10

\section{Introduction}

The thickness $\theta(G)$ of a graph $G$ is the minimum number of planar subgraphs whose union is $G$. It is a measurement of the planarity of a graph, the graph with $\theta(G)=1$ is a planar graph; it also has important application in VLSI design [15]. Since W. T. Tutte [16] inaugurated the thickness problem in 1963, the thickness of some classic types of graphs have been obtained by various authors, such as [1, 3, 4, 13, 17, 19] etc. In recent years, some authors focus on the thickness of the graphs which are obtained by operating on two graphs, such as the Cartesian product graph [8, 20] and join graph [7]. In this paper, we are concerned with the Kronecker product graph.

* Supported by the National Natural Science Foundation of China under Grant No. 11401430. The authors are grateful to Bojan Mohar for helpful comments after the second author gave a talk on this topic in Beijing, March 2019. Especially, Bojan Mohar helped us to state the upper bound in Theorem 2.1 in an improved form. The authors also thank the referees for their helpful comments and suggestions.

${ }^{\dagger}$ Corresponding author.

E-mail addresses: guoxia@stu.xmu.edu.cn (Xia Guo), yanyang@tju.edu.cn (Yan Yang)

()( This work is licensed under https://creativecommons.org/licenses/by/4.0/ 
The Kronecker product (also called as tensor product, direct product, categorical product) $G \times H$ of graphs $G$ and $H$ is the graph whose vertex set is $V(G \times H)=V(G) \times V(H)$ and edge set is $E(G \times H)=\left\{(g, h)\left(g^{\prime}, h^{\prime}\right) \mid g g^{\prime} \in E(G)\right.$ and $\left.h h^{\prime} \in E(H)\right\}$. Figure 1 shows the Kronecker product graph $K_{5} \times K_{2}$ in which $\left\{u_{1}, \ldots, u_{5}\right\}$ and $\left\{v_{1}, v_{2}\right\}$ are the vertex sets of the complete graphs $K_{5}$ and $K_{2}$, respectively. Many authors did research on various topics of the Kronecker product graph, such as for its planarity [2,10], connectivity [18], coloring [9, 12] and application [14] etc.

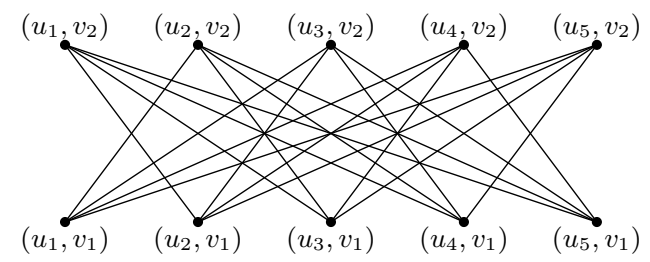

Figure 1: The Kronecker product graph $K_{5} \times K_{2}$.

The complete graph $K_{n}$ is the graph on $n$ vertices in which any two vertices are adjacent. The complete bipartite graph $K_{m, n}$ is the graph whose vertex set can be partitioned into two parts $X$ and $Y,|X|=m$ and $|Y|=n$, every edge has its ends in different parts and every two vertices in different parts are adjacent. The complete tripartite graph $K_{l, m, n}$ is defined analogously.

In this paper, we present lower and upper bounds for the thickness of the Kronecker product of two graphs in Section 2, in which the lower bound comes from Euler's formula and the upper bound is derived from the structure of the Kronecker product graph. Then we study the thickness of the Kronecker product of a graph with $K_{2}$. There are two reasons why we interested in it. One reason is that the upper bound for the thickness of the Kronecker product of two graphs we will provide relies on that of the Kronecker product of a graph with $K_{2}$. Another reason is that the planarity of the Kronecker product of two graphs have been characterized in [10], but a graph with $K_{2}$ is one of its missing cases. It's a difficult case, because there exist non-planar graphs whose Kronecker product with $K_{2}$ are planar graphs, see Figures 1 and 2 in [2] for example. In Sections 3 and 4, we provide the exact thickness numbers for the Kronecker product graphs $K_{n} \times K_{2}, K_{m, n} \times K_{2}$ and $K_{n, n, n} \times K_{2}$.

For undefined terminology, see [5].

\section{Thickness of the Kronecker product graph $\boldsymbol{G} \times \boldsymbol{H}$}

A $k$-edge-coloring of a graph $G$ is a mapping $f: E(G) \rightarrow S$, where $S$ is a set of $k$ colors. A $k$-edge-coloring is proper if incident edges have different colors. A graph is $k$-edgecolorable if it has a proper $k$-edge-coloring. The edge chromatic number $\chi^{\prime}(G)$ of a graph $G$ is the least $k$ such that $G$ is $k$-edge-colorable.

Theorem 2.1. Let $G$ and $H$ be two simple graphs on at least two vertices, then

$$
\left\lceil\frac{2|E(G)||E(H)|}{3|V(G)||V(H)|-6}\right\rceil \leq \theta(G \times H) \leq \min \left\{\chi^{\prime}(H) \theta\left(G \times K_{2}\right), \chi^{\prime}(G) \theta\left(H \times K_{2}\right)\right\},
$$

in which $\chi^{\prime}(H)$ and $\chi^{\prime}(G)$ are edge chromatic number of $H$ and $G$ respectively. 
Proof. It is easy to observe that the number of edges in $G \times H$ is $|E(G \times H)|=$ $2|E(G)||E(H)|$ and the number of vertices in $G \times H$ is $|V(G \times H)|=|V(G)||V(H)|$. From the Euler's Formula, the planar graph with $|V(G)||V(H)|$ vertices, has at most $3|V(G)||V(H)|-6$ edges, the lower bound follows.

The $\chi^{\prime}(H)$-edge-coloring of $H$ can be seen as a partition $\left\{M_{1}, \ldots, M_{\chi^{\prime}(H)}\right\}$ of $E(H)$, in which $M_{i}$ denotes the set of edges assigned color $i\left(1 \leq i \leq \chi^{\prime}(H)\right)$. Then $M_{i}$ is a matching and $E(H)=M_{1} \cup \cdots \cup M_{\chi^{\prime}(H)}$. Because $G \times H=\cup_{i=1}^{\chi^{\prime}(H)}\left(G \times M_{i}\right)$ and $\theta\left(G \times M_{i}\right)=\theta\left(G \times K_{2}\right)$, we have $\theta(G \times H) \leq \chi^{\prime}(H) \theta\left(G \times K_{2}\right)$. With the same argument, we have $\theta(G \times H) \leq \chi^{\prime}(G) \theta\left(H \times K_{2}\right)$. The upper bound can be derived.

In the following, we will give examples to show both the lower and upper bound in Theorem 2.1 are sharp. Let $G$ and $H$ be the graphs as shown in Figure 2(a) and (b) respectively. Figure 2(c) illustrates a planar embedding of the graph $G \times\left\{v_{1} v_{2}\right\}$, in which we denote the vertex $\left(u_{i}, v_{j}\right)$ by $u_{i}^{j}, 1 \leq i \leq 7,1 \leq j \leq 2$. So the thickness of $G \times\left\{v_{1} v_{2}\right\}$ is one which meets the lower bound in Theorem 2.1. Figure 2(d) illustrates a planar embedding of the graph $G \times\left\{v_{2} v_{3}\right\}$ which is isomorphic to $G \times\left\{v_{1} v_{2}\right\}$. Because $G \times H=G \times\left\{v_{1} v_{2}\right\} \cup G \times\left\{v_{2} v_{3}\right\}$, we get a planar subgraph decomposition of $G \times H$ with two subgraphs, which shows the thickness of $G \times H$ is not more than two. On the other hand, the graph $G \times H$ contains a subdivision of $K_{5}$ which is exhibited in Figure 2(e), so $G \times H$ is not a planar graph, its thickness is greater than one. Therefore, the thickness of $G \times H$ is two which meets the upper bound in Theorem 2.1.

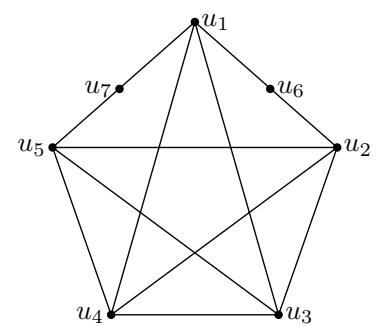

(a) The graph $G$.

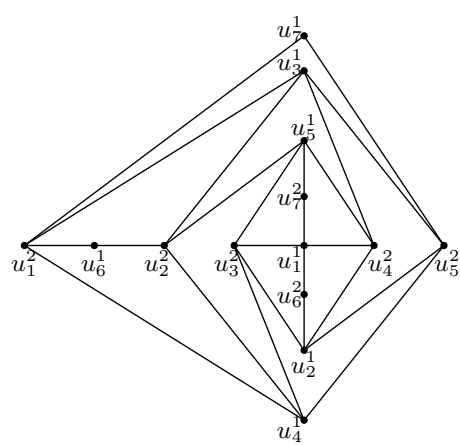

(c) The graph $G \times\left\{v_{1} v_{2}\right\}$.

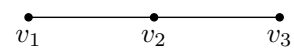

(b) The graph $H$.

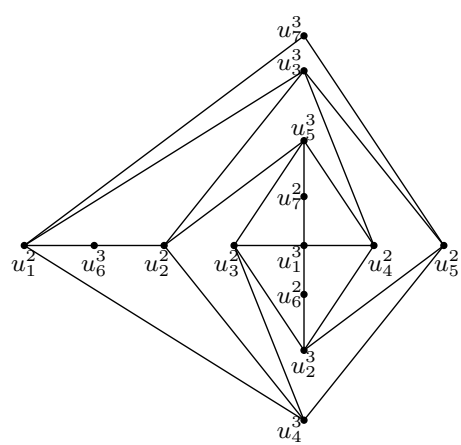

(d) The graph $G \times\left\{v_{2} v_{3}\right\}$.

Figure 2: An example to show both lower and upper bounds in Theorem 2.1 are sharp. 


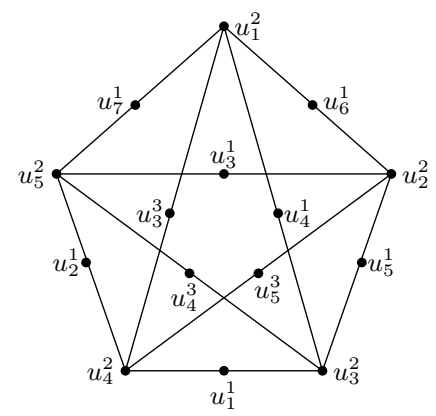

(e) A subgraph of $G \times H$.

Figure 2: An example to show both lower and upper bounds in Theorem 2.1 are sharp.

The graph $G \times H$ has a triangle if and only if both $G$ and $H$ have triangles. If $G \times H$ does not contain any triangles, from the Euler's Formula, the planar graph with $|V(G)||V(H)|$ vertices, has at most $2|V(G)||V(H)|-4$ edges, a tighter lower bound can be derived.

Theorem 2.2. Let $G$ and $H$ be two simple graphs on at least two vertices. If $G \times H$ does not contain any triangles, then

$$
\left\lceil\frac{|E(G)||E(H)|}{|V(G)||V(H)|-2}\right\rceil \leq \theta(G \times H) \leq \min \left\{\chi^{\prime}(H) \theta\left(G \times K_{2}\right), \chi^{\prime}(G) \theta\left(H \times K_{2}\right)\right\} .
$$

\section{The thickness of $K_{n} \times K_{2}$ and $K_{m, n} \times K_{2}$}

In this section, by making use of the thickness number of $K_{n, n}$ and a known planar decomposition of $K_{n, n}$ as shown in Lemmas 3.1 and 3.2 respectively, we will obtain the exact thickness numbers of $K_{n} \times K_{2}$ and $K_{m, n} \times K_{2}$.

Let $G$ be a simple graph with $n$ vertices, $V(G)=\left\{v_{1}, \ldots, v_{n}\right\}$ and $V\left(K_{2}\right)=\{1,2\}$. Then $G \times K_{2}$ is a bipartite graph, the two vertex parts are $\left\{\left(v_{i}, 1\right) \mid 1 \leq i \leq n\right\}$ and $\left\{\left(v_{i}, 2\right) \mid 1 \leq i \leq n\right\}$, so $G \times K_{2}$ is a subgraph of $K_{n, n}$ which shows that $\theta\left(G \times K_{2}\right) \leq$ $\theta\left(K_{n, n}\right)$. Although the thickness of the complete bipartite $K_{m, n}$ have not been solved completely, when $m=n$, the following result is known.

Lemma 3.1 ([4]). The thickness of the complete bipartite graph $K_{n, n}$ is

$$
\theta\left(K_{n, n}\right)=\left\lceil\frac{n+2}{4}\right\rceil
$$

When $n=4 p(p \geq 1)$, Chen and Yin [8] gave a planar subgraphs decomposition of $K_{4 p, 4 p}$ with $p+1$ planar subgraphs $G_{1}, \ldots, G_{p+1}$. Denote the two vertex parts of $K_{4 p, 4 p}$ by $U=\left\{u_{1}, \ldots, u_{4 p}\right\}$ and $V=\left\{v_{1}, \ldots, v_{4 p}\right\}$, Figure 3 shows their planar subgraphs decomposition of $K_{4 p, 4 p}$, in which for each $G_{r}(1 \leq r \leq p)$, both $v_{4 r-3}$ and $v_{4 r-1}$ join to each vertex in set $\bigcup_{i=1, i \neq r}^{p}\left\{u_{4 i-3}, u_{4 i-2}\right\}$, both $v_{4 r-2}$ and $v_{4 r}$ join to each vertex in set $\bigcup_{i=1, i \neq r}^{p}\left\{u_{4 i-1}, u_{4 i}\right\}$, both $u_{4 r-1}$ and $u_{4 r}$ join to each vertex in set $\bigcup_{i=1, i \neq r}^{p}\left\{v_{4 i-3}, v_{4 i-1}\right\}$, and both $u_{4 r-3}$ and $u_{4 r-2}$ join to each vertex in set $\bigcup_{i=1, i \neq r}^{p}\left\{v_{4 i-2}, v_{4 i}\right\}$. Notice that $G_{p+1}$ is a perfect matching of $K_{4 p, 4 p}$, the edge set of it is $\left\{u_{i} v_{i} \mid 1 \leq i \leq 4 p\right\}$. 


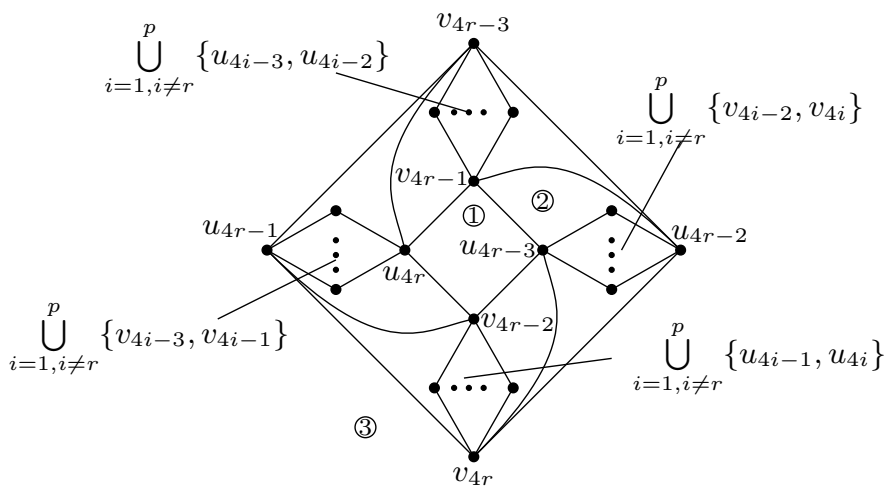

(a) The graph $G_{r}(1 \leq r \leq p)$.

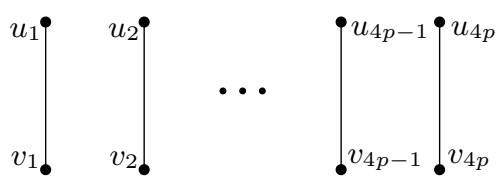

(b) The graph $G_{p+1}$.

Figure 3: A planar decomposition of $K_{4 p, 4 p}$.

Lemma 3.2 ([8]). Suppose $K_{n, n}$ is a complete bipartite graph with two vertex parts $U=$ $\left\{u_{1}, \ldots, u_{n}\right\}$ and $V=\left\{v_{1}, \ldots, v_{n}\right\}$. When $n=4 p$, there exists a planar subgraphs decomposition of $K_{4 p, 4 p}$ with $p+1$ planar subgraphs $G_{1}, \ldots, G_{p+1}$ in which $G_{p+1}$ is a perfect matching of $K_{4 p, 4 p}$ with edge set $\left\{u_{i} v_{i} \mid 1 \leq i \leq 4 p\right\}$.

Theorem 3.3. The thickness of the Kronecker product of $K_{n}$ and $K_{2}$ is

$$
\theta\left(K_{n} \times K_{2}\right)=\left\lceil\frac{n}{4}\right\rceil .
$$

Proof. Suppose that the vertex sets of $K_{n}$ and $K_{2}$ are $\left\{x_{1}, \ldots, x_{n}\right\}$ and $\{1,2\}$ respectively. The graph $K_{n} \times K_{2}$ is a bipartite graph whose two vertex parts are $\left\{\left(x_{i}, 1\right) \mid 1 \leq i \leq n\right\}$ and $\left\{\left(x_{i}, 2\right) \mid 1 \leq i \leq n\right\}$, and edge set is $\left\{\left(x_{i}, 1\right)\left(x_{j}, 2\right) \mid 1 \leq i, j \leq n, i \neq j\right\}$. For $1 \leq i \leq n, 1 \leq k \leq 2$, we denote the vertex $\left(x_{i}, k\right)$ of $K_{n} \times K_{2}$ by $x_{i}^{k}$ for simplicity. have

Since $\left|E\left(K_{n} \times K_{2}\right)\right|=n(n-1)$ and $\left|V\left(K_{n} \times K_{2}\right)\right|=2 n$, from Theorem 2.2, we

$$
\theta\left(K_{n} \times K_{2}\right) \geq\left\lceil\frac{n(n-1)}{4 n-4}\right\rceil=\left\lceil\frac{n}{4}\right\rceil .
$$

In the following, we will construct planar decompositions of $K_{n} \times K_{2}$ with $\left\lceil\frac{n}{4}\right\rceil$ subgraphs to complete the proof.

Case 1. When $n=4 p$.

Suppose that $K_{n, n}$ is a complete bipartite graph with vertex partition $\left(X^{1}, X^{2}\right)$ in which $X^{1}=\left\{x_{1}^{1}, \ldots, x_{n}^{1}\right\}$ and $X^{2}=\left\{x_{1}^{2}, \ldots, x_{n}^{2}\right\}$. The graph $G_{p+1}$ is a perfect matching of $K_{4 p, 4 p}$ whose edge set is $\left\{x_{i}^{1} x_{i}^{2} \mid 1 \leq i \leq n\right\}$, then $K_{n} \times K_{2}=K_{n, n}-G_{p+1}$. From Lemma 3.2, there exists a planar decomposition $\left\{G_{1}, \ldots, G_{p}\right\}$ of $K_{n} \times K_{2}$ in which $G_{r}$ $(1 \leq r \leq p)$ is isomorphic to the graph in Figure 3(a). Therefore, $\theta\left(K_{4 p} \times K_{2}\right) \leq p$. 
Case 2. When $n=4 p+2$.

When $p \geq 1$, we draw a graph $G_{p+1}^{\prime}$ as shown in Figure 4 , then $\left\{G_{1}, \ldots, G_{p}, G_{p+1}^{\prime}\right\}$ is a planar decomposition of $K_{4 p+2} \times K_{2}$ with $p+1$ subgraphs, so we have $\theta\left(K_{4 p+2} \times K_{2}\right) \leq$ $p+1$. When $n=2, K_{2} \times K_{2}=2 K_{2}$ is a planar graph.

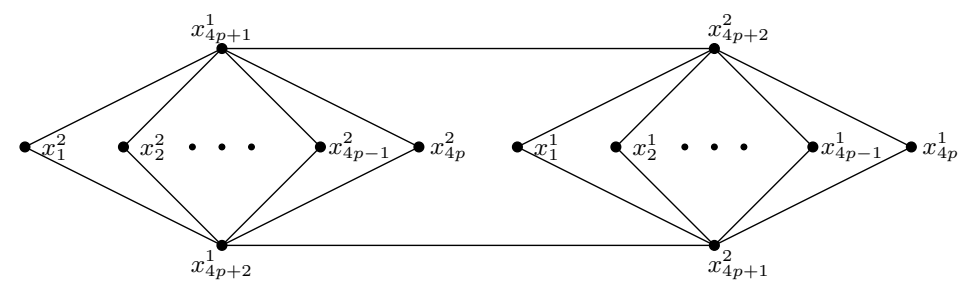

Figure 4: The graph $G_{p+1}^{\prime}$.

Case 3. When $n=4 p+1$ and $n=4 p+3$.

Because $K_{4 p+1} \times K_{2}$ is a subgraph of $K_{4 p+2} \times K_{2}$, we have $\theta\left(K_{4 p+1} \times K_{2}\right) \leq$ $\theta\left(K_{4 p+2} \times K_{2}\right)=p+1$. Similarly, when $n=4 p+3$, we have $\theta\left(K_{4 p+3} \times K_{2}\right) \leq$ $\theta\left(K_{4(p+1)} \times K_{2}\right)=p+1$.

Summarizing Cases 1, 2 and 3, we have

$$
\theta\left(K_{n} \times K_{2}\right) \leq\left\lceil\frac{n}{4}\right\rceil .
$$

Theorem follows from inequalities (3.1) and (3.2).

Theorem 3.4. Let $G$ be a simple graph on $n(n \geq 2)$ vertices, then

$$
\left\lceil\frac{E(G)}{2 n-2}\right\rceil \leq \theta\left(G \times K_{2}\right) \leq\left\lceil\frac{n}{4}\right\rceil .
$$

Proof. Because $G \times K_{2}$ is a subgraph of $K_{n} \times K_{2}$, we have $\theta\left(G \times K_{2}\right) \leq \theta\left(K_{n} \times K_{2}\right)$. Combining it with Theorems 2.2 and 3.3, the theorem follows.

Lemma 3.5 ([10]). The Kronecker product of $K_{m, n}$ and $K_{p, q}$ is a disjoint union $K_{m p, n q} \cup$ $K_{m q, n p}$.

Theorem 3.6. The thickness of the Kronecker product of $K_{m, n}$ and $K_{p, q}$ is

$$
\theta\left(K_{m, n} \times K_{p, q}\right)=\max \left\{\theta\left(K_{m p, n q}\right), \theta\left(K_{m q, n p}\right)\right\} .
$$

Proof. From Lemma 3.5, the proof is straightforward.

Because $K_{2}$ is also $K_{1,1}$, the following corollaries are easy to get, from Theorem 3.6 and Lemma 3.1.

Corollary 3.7. The thickness of the Kronecker product of $K_{m, n}$ and $K_{2}$ is

$$
\theta\left(K_{m, n} \times K_{2}\right)=\theta\left(K_{m, n}\right) .
$$

Corollary 3.8. The thickness of the Kronecker product of $K_{n, n}$ and $K_{2}$ is

$$
\theta\left(K_{n, n} \times K_{2}\right)=\left\lceil\frac{n+2}{4}\right\rceil .
$$




\section{The thickness of the Kronecker product graph $K_{n, n, n} \times K_{2}$}

Let $(X, Y, Z)$ be the vertex partition of the complete tripartite graph $K_{l, m, n}(l \leq m \leq n)$ in which $X=\left\{x_{1}, \ldots, x_{l}\right\}, Y=\left\{y_{1}, \ldots, y_{m}\right\}, Z=\left\{z_{1}, \ldots, z_{n}\right\}$. Let $\{1,2\}$ be the vertex set of $K_{2}$. We denote the vertex $(v, k)$ of $K_{l, m, n} \times K_{2}$ by $v^{k}$ in which $v \in V\left(K_{l, m, n}\right)$ and $k \in\{1,2\}$. For $k=1,2$, we denote $X^{k}=\left\{x_{1}^{k}, \ldots, x_{l}^{k}\right\}, Y^{k}=\left\{y_{1}^{k}, \ldots, y_{m}^{k}\right\}$ and $Z^{k}=\left\{z_{1}^{k}, \ldots, z_{n}^{k}\right\}$. In Figure 5, we draw a sketch of the graph $K_{l, m, n} \times K_{2}$, in which the edge joining two vertex set indicates that each vertex in one vertex set is adjacent to each vertex in another vertex set. Suppose $G\left(X^{1}, Y^{2}\right)$ is the graph induced by the vertex sets $X^{1}$ and $Y^{2}$ of $K_{l, m, n} \times K_{2}$, then $G\left(X^{1}, Y^{2}\right)$ is isomorphic to $K_{l, m}$, the graphs $G\left(Y^{1}, Z^{2}\right)$, $G\left(Z^{1}, X^{2}\right), G\left(X^{2}, Y^{1}\right), G\left(Y^{2}, Z^{1}\right)$ and $G\left(Z^{2}, X^{1}\right)$ are defined analogously. We define

$$
G^{1}=G\left(X^{1}, Y^{2}\right) \cup G\left(Y^{1}, Z^{2}\right) \cup G\left(Z^{1}, X^{2}\right)
$$

and

$$
G^{2}=G\left(X^{2}, Y^{1}\right) \cup G\left(Y^{2}, Z^{1}\right) \cup G\left(Z^{2}, X^{1}\right),
$$

then $K_{l, m, n} \times K_{2}=G^{1} \cup G^{2}$.

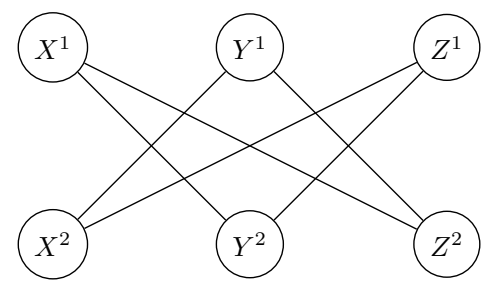

Figure 5: The graph $K_{l, m, n} \times K_{2}$.

Theorem 4.1. The thickness of the Kronecker product graph $K_{l, m, n} \times K_{2}(l \leq m \leq n)$ satisfies the inequality

$$
\left\lceil\frac{l m+l n+m n}{2(l+m+n)-2}\right\rceil \leq \theta\left(K_{l, m, n} \times K_{2}\right) \leq 2 \theta\left(K_{m, n}\right) .
$$

Proof. From Theorem 3.4, one can get the lower bound in this theorem easily. Any two graphs of $G\left(X^{1}, Y^{2}\right), G\left(Y^{1}, Z^{2}\right)$ and $G\left(Z^{1}, X^{2}\right)$ are disjoint with each other and $l \leq$ $m \leq n$, so we have

$$
\theta\left(G^{1}\right) \leq \max \left\{\theta \left(G\left(X^{1}, Y^{2}\right), \theta\left(G\left(Y^{1}, Z^{2}\right), \theta\left(G\left(Z^{1}, X^{2}\right)\right\}=\theta\left(K_{m, n}\right) .\right.\right.\right.
$$

Similarly, we have

$$
\theta\left(G^{2}\right) \leq \max \left\{\theta \left(G\left(X^{2}, Y^{1}\right), \theta\left(G\left(Y^{2}, Z^{1}\right), \theta\left(G\left(Z^{2}, X^{1}\right)\right)\right\}=\theta\left(K_{m, n}\right) .\right.\right.
$$

Due to the graph $K_{l, m, n} \times K_{2}=G^{1} \cup G^{2}$, we have $\theta\left(K_{l, m, n} \times K_{2}\right) \leq 2 \theta\left(K_{m, n}\right)$. Summarizing the above, the theorem is obtained.

In the following, we will construct planar decompositions of $K_{n, n, n} \times K_{2}$ when $n=$ $4 p, 4 p+1,4 p+3$ in Lemmas 4.2, 4.4 and 4.5 respectively. Then combining these lemmas with Theorem 2.2, we will get the thickness of $K_{n, n, n} \times K_{2}$ and we will see when $n=$ $4 p+2$, the upper and lower bound in Theorem 4.1 are equal, so both bounds in Theorem 4.1 are sharp. 
Lemma 4.2. When $n=4 p$, there exists a planar decomposition of the Kronecker product graph $K_{n, n, n} \times K_{2}$ with $2 p+1$ subgraphs.

Proof. Because $\left|X^{k}\right|=\left|Y^{k}\right|=\left|Z^{k}\right|=n(k=1,2)$, all the graphs $G\left(X^{1}, Y^{2}\right)$, $G\left(Y^{1}, Z^{2}\right), G\left(Z^{1}, X^{2}\right), G\left(X^{2}, Y^{1}\right), G\left(Y^{2}, Z^{1}\right), G\left(Z^{2}, X^{1}\right)$ are isomorphic to $K_{n, n}$.

Let $\left\{G_{1}, \ldots, G_{p+1}\right\}$ be the planar decomposition of $K_{n, n}$ as shown in Figure 3. For $1 \leq r \leq p+1, G_{r}$ is a bipartite graph, so we also denote it by $G_{r}(V, U)$. In $G_{r}(V, U)$, we replace the vertex set $V$ by $X^{1}, U$ by $Y^{2}$, i.e., for each $1 \leq i \leq n$, replace the vertex $v_{i}$ by $x_{i}^{1}$, and $u_{i}$ by $y_{i}^{2}$, then we get graph $G_{r}\left(X^{1}, Y^{2}\right)$. Analogously, we obtain graphs $G_{r}\left(Y^{1}, Z^{2}\right), G_{r}\left(Z^{1}, X^{2}\right), G_{r}\left(X^{2}, Y^{1}\right), G_{r}\left(Y^{2}, Z^{1}\right)$ and $G_{r}\left(Z^{2}, X^{1}\right)$.

For $1 \leq r \leq p+1$, let

$$
G_{r}^{1}=G_{r}\left(X^{1}, Y^{2}\right) \cup G_{r}\left(Y^{1}, Z^{2}\right) \cup G_{r}\left(Z^{1}, X^{2}\right)
$$

and

$$
G_{r}^{2}=G_{r}\left(X^{2}, Y^{1}\right) \cup G_{r}\left(Y^{2}, Z^{1}\right) \cup G_{r}\left(Z^{2}, X^{1}\right) .
$$

Because $G_{r}\left(X^{1}, Y^{2}\right), G_{r}\left(Y^{1}, Z^{2}\right), G_{r}\left(Z^{1}, X^{2}\right)$ are all planar graphs and they are disjoint with each other, $G_{r}^{1}$ is a planar graph. For the same reason, we have that $G_{r}^{2}$ is also a planar graph.

Let graph $G_{p+1}$ be the graph $G_{p+1}^{1} \cup G_{p+1}^{2}$. We have

$$
\begin{aligned}
G_{p+1} & =G_{p+1}^{1} \cup G_{p+1}^{2} \\
& =\left\{\bigcup_{i=1}^{n}\left(x_{i}^{1} y_{i}^{2} \cup y_{i}^{1} z_{i}^{2} \cup z_{i}^{1} x_{i}^{2}\right)\right\} \cup\left\{\bigcup_{i=1}^{n}\left(x_{i}^{2} y_{i}^{1} \cup y_{i}^{2} z_{i}^{1} \cup z_{i}^{2} x_{i}^{1}\right)\right\} \\
& =\bigcup_{i=1}^{n}\left(x_{i}^{1} y_{i}^{2} z_{i}^{1} x_{i}^{2} y_{i}^{1} z_{i}^{2} x_{i}^{1}\right) .
\end{aligned}
$$

It is easy to see $G_{p+1}$ consists of $n$ disjoint cycles of length 6 , hence $G_{p+1}$ is a planar graph.

Because

$$
\begin{aligned}
G\left(X^{1}, Y^{2}\right) & =\bigcup_{r=1}^{p+1} G_{r}\left(X^{1}, Y^{2}\right), & G\left(Y^{1}, Z^{2}\right) & =\bigcup_{r=1}^{p+1} G_{r}\left(Y^{1}, Z^{2}\right), \\
G\left(Z^{1}, X^{2}\right) & =\bigcup_{r=1}^{p+1} G_{r}\left(Z^{1}, X^{2}\right), & G\left(X^{2}, Y^{1}\right) & =\bigcup_{r=1}^{p+1} G_{r}\left(X^{2}, Y^{1}\right),
\end{aligned}
$$

and

$$
G\left(Y^{2}, Z^{1}\right)=\bigcup_{r=1}^{p+1} G_{r}\left(Y^{2}, Z^{1}\right), \quad G\left(Z^{2}, X^{1}\right)=\bigcup_{r=1}^{p+1} G_{r}\left(Z^{2}, X^{1}\right),
$$

we have

$$
\begin{aligned}
K_{n, n, n} \times K_{2} & =G^{1} \cup G^{2} \\
& =\bigcup_{r=1}^{p+1}\left(G_{r}^{1} \cup G_{r}^{2}\right) \\
& =\bigcup_{r=1}^{p}\left(G_{r}^{1} \cup G_{r}^{2}\right) \cup G_{p+1} .
\end{aligned}
$$

So we get a planar decomposition of $K_{4 p, 4 p, 4 p} \times K_{2}$ with $2 p+1$ subgraphs $G_{1}^{1}, \ldots, G_{p}^{1}$, $G_{1}^{2}, \ldots, G_{p}^{2}, G_{p+1}$. The proof is completed. 
We draw the planar decomposition of $K_{8,8,8} \times K_{2}$ as shown in Figure 6 .

Lemma 4.3 ([5]). Let $G$ be a planar graph, and let $f$ be a face in some planar embedding of $G$. Then $G$ admits a planar embedding whose outer face has the same boundary as $f$.
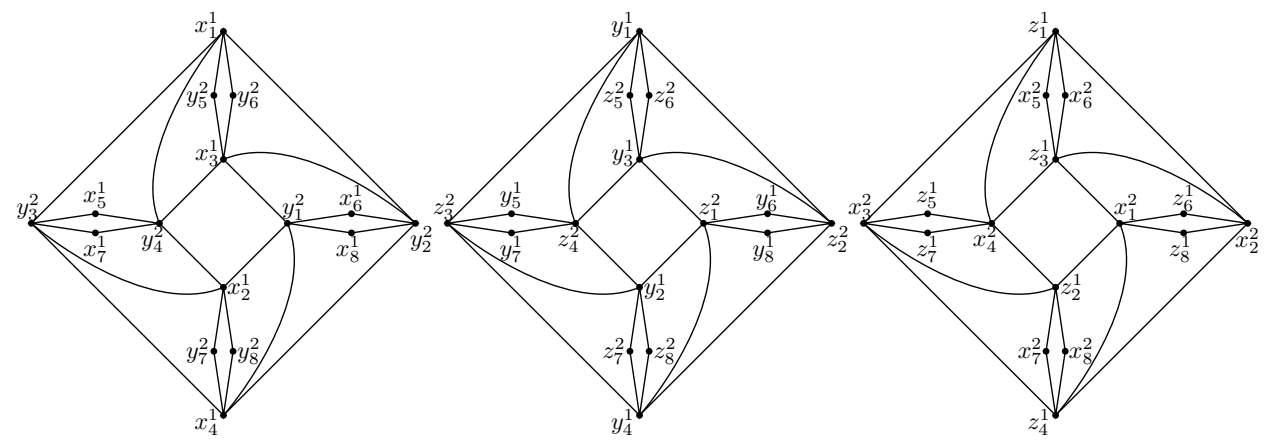

(a) The graph $G_{1}^{1}$.
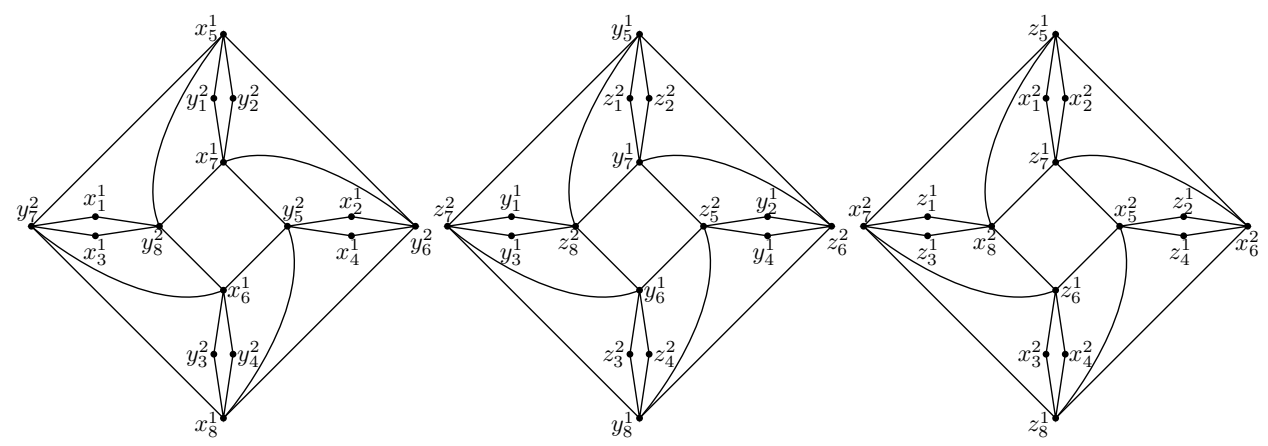

(b) The graph $G_{2}^{1}$.
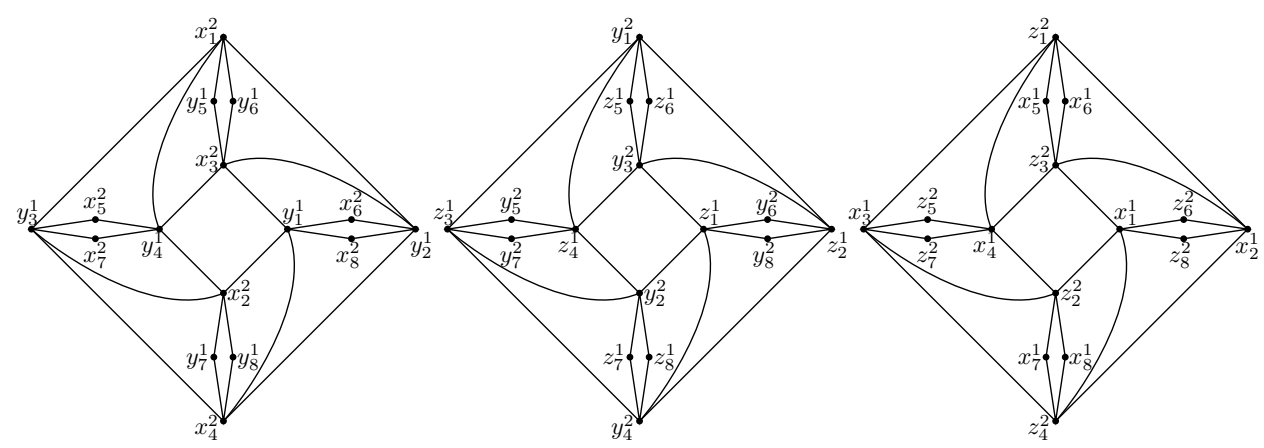

(c) The graph $G_{1}^{2}$.

Figure 6: A planar decomposition of $K_{8,8,8} \times K_{2}$. 

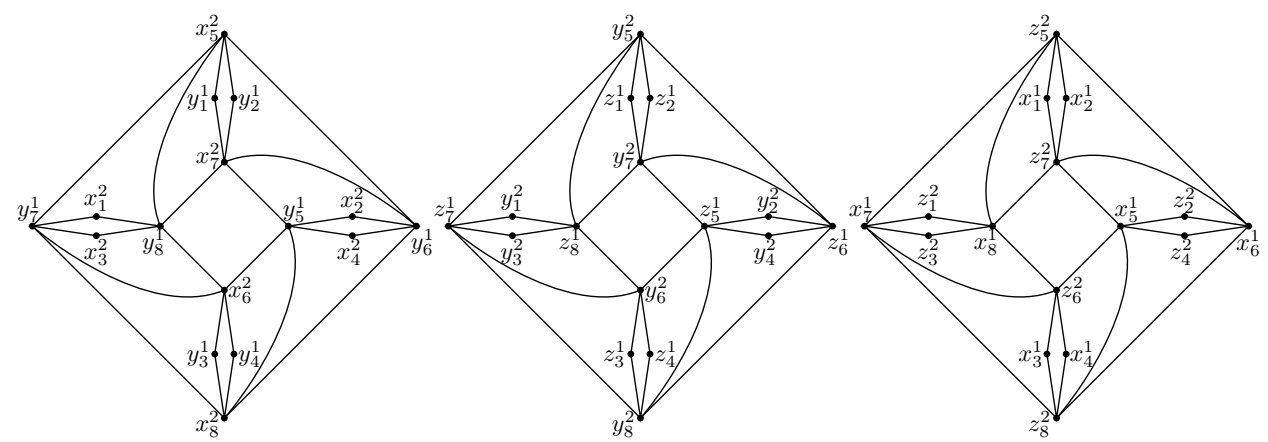

(d) The graph $G_{2}^{2}$.
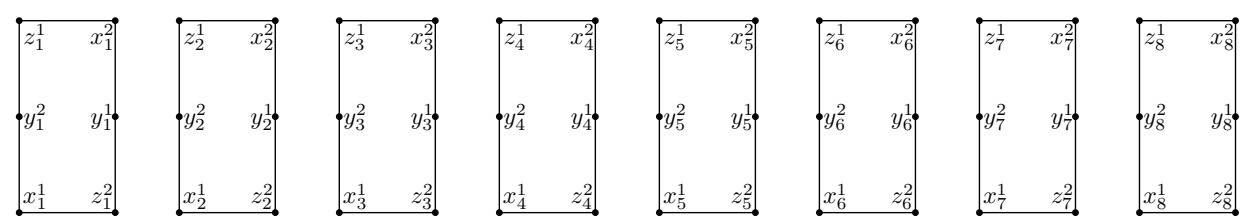

(e) The graph $G_{3}$.

Figure 6: A planar decomposition of $K_{8,8,8} \times K_{2}$.

Lemma 4.4. When $n=4 p+1$, there exists a planar decomposition of the Kronecker product graph $K_{n, n, n} \times K_{2}$ with $2 p+1$ subgraphs.

Proof. Case 1. When $p \leq 1$.

When $p=0$, the Kronecker product graph $K_{1,1,1} \times K_{2}$ is a cycle of length 6 , so $K_{1,1,1} \times K_{2}$ is a planar graph. When $p=1$, as shown in Figure 7, we give a planar decomposition of $K_{5,5,5} \times K_{2}$ with three subgraphs $A, B$ and $C$.

Case 2. When $p \geq 2$.

Suppose that $\left\{G_{1}^{1}, \ldots, G_{p}^{1}, G_{1}^{2}, \ldots, G_{p}^{2}, G_{p+1}\right\}$ is the planar decomposition of $K_{4 p, 4 p, 4 p} \times$ $K_{2}$ as provided in the proof of Lemma 4.2. By adding vertices $x_{4 p+1}^{1}, x_{4 p+1}^{2}, y_{4 p+1}^{1}, y_{4 p+1}^{2}$, $z_{4 p+1}^{1}, z_{4 p+1}^{2}$ to each graph in this decomposition, and some modifications of adding and deleting edges to these graphs, a planar decomposition of $K_{4 p+1,4 p+1,4 p+1} \times K_{2}$ will be obtained.

For convenience, in Figure 3(a) we label some faces of $G_{r}(1 \leq r \leq p)$ with face 1,2 and 3. As indicated in Figure 3(a), the face 1 is bounded by $v_{4 r-1} u_{4 r-3} v_{4 r-2} u_{4 r}$, the face 3 is its outer face, bounded by $v_{4 r-3} u_{4 r-2} v_{4 r} u_{4 r-1}$. The face 2 is bounded by $u_{4 r-3} v_{4 r-1} u_{4 r-2} v_{j}$ in which vertex $v_{j}$ can be any vertex of $\bigcup_{i=1, i \neq r}^{p}\left\{v_{4 i-2}, v_{4 i}\right\}$. Because $u_{4 r-3}$ and $u_{4 r-2}$ in $G_{r}(1 \leq r \leq p)$ is joined by $2 p-2$ edge-disjoint paths of length two that we call parallel paths, we can change the order of these parallel paths without changing the planarity of $G_{r}$. Analogously, we can change the order of parallel paths between $u_{4 r-1}$ and $u_{4 r}, v_{4 r-3}$ and $v_{4 r-1}, v_{4 r-2}$ and $v_{4 r}$. In addition, the subscripts of all the vertices are taken module $4 p$, except that of the new added vertices $x_{4 p+1}^{1}, x_{4 p+1}^{2}, y_{4 p+1}^{1}, y_{4 p+1}^{2}, z_{4 p+1}^{1}$ and $z_{4 p+1}^{2}$. 


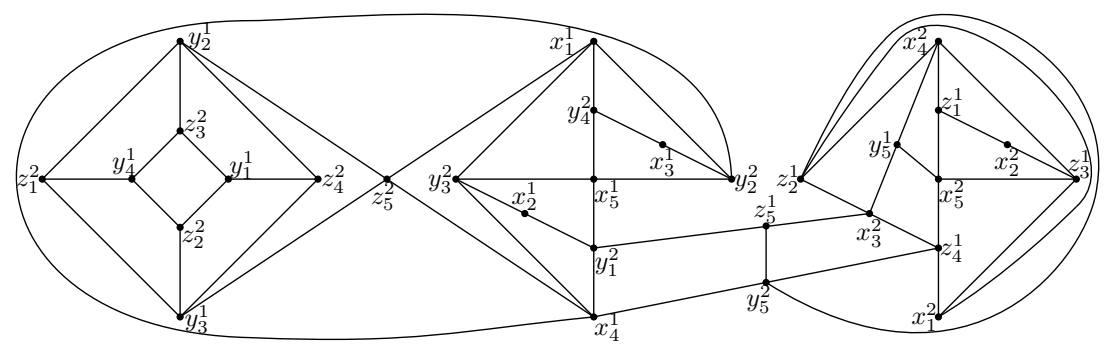

(a) The graph $A$.

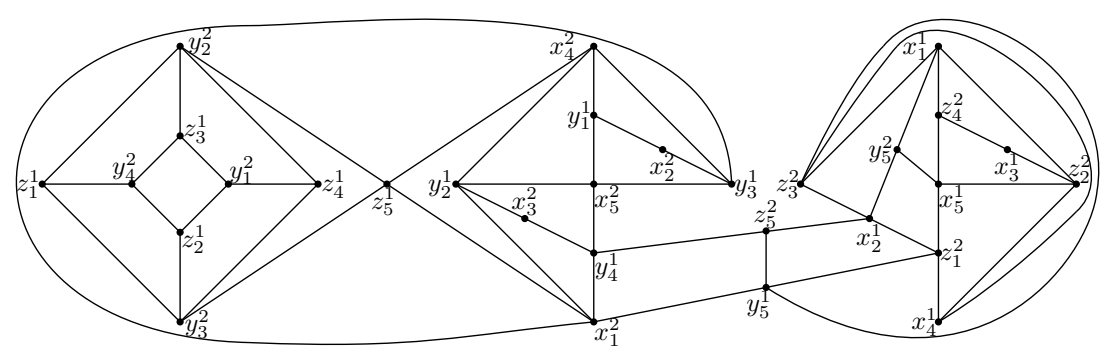

(b) The graph $B$.
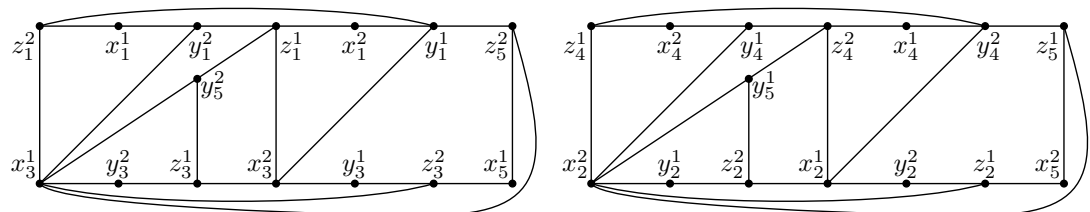

(c) The graph $C$.

Figure 7: A planar decomposition of $K_{5,5,5} \times K_{2}$.

Step 1: Add the vertices $x_{4 p+1}^{1}$ and $y_{4 p+1}^{2}$ to graph $G_{r}\left(X^{1}, Y^{2}\right)$.

Place vertices $x_{4 p+1}^{1}$ and $y_{4 p+1}^{2}$ in face 1 and face 2 of $G_{r}\left(X^{1}, Y^{2}\right)$, respectively. Join $x_{4 p+1}^{1}$ to vertices $y_{4 r-3}^{2}$ and $y_{4 r}^{2}$. Change the order of the parallel paths between $y_{4 r-2}^{2}$ and $y_{4 r-3}^{2}$, such that $x_{4 r+2}^{1} \in \bigcup_{i=1, i \neq r}^{p}\left\{x_{4 i-2}^{1}, x_{4 i}^{1}\right\}$ are incident with the face 2 , and join $y_{4 p+1}^{2}$ to both $x_{4 r-1}^{1}$ and $x_{4 r+2}^{1}$.

Step 2: Add the vertices $x_{4 p+1}^{2}$ and $y_{4 p+1}^{1}$ to graph $G_{r}\left(X^{2}, Y^{1}\right)$.

Similar to step 1, place $x_{4 p+1}^{2}$ and $y_{4 p+1}^{1}$ in face 1 and face 2 of $G_{r}\left(X^{2}, Y^{1}\right)$, respectively. Join $x_{4 p+1}^{2}$ to both $y_{4 r-3}^{1}$ and $y_{4 r}^{1}$, join $y_{4 p+1}^{1}$ to both $x_{4 r-1}^{2}$ and $x_{4 r+2}^{2} \in \bigcup_{i=1, i \neq r}^{p}\left\{x_{4 i-2}^{2}\right.$, $\left.x_{4 i}^{2}\right\}$. 
Step 3: Add the vertices $y_{4 p+1}^{1}$ and $z_{4 p+1}^{2}$ to graph $G_{r}\left(Y^{1}, Z^{2}\right)$.

Place $y_{4 p+1}^{1}$ in face 3 of $G_{r}\left(Y^{1}, Z^{2}\right)$ and join it to vertices $z_{4 r-2}^{2}$ and $z_{4 r-1}^{2}$. Place $z_{4 p+1}^{2}$ in face 1 of $G_{r}\left(Y^{1}, Z^{2}\right)$ and join it to vertices $y_{4 r-2}^{1}$ and $y_{4 r-1}^{1}$.

Step 4: Add the vertices $y_{4 p+1}^{2}$ and $z_{4 p+1}^{1}$ to graph $G_{r}\left(Y^{2}, Z^{1}\right)$.

Place $y_{4 p+1}^{2}$ in face 3 of $G_{r}\left(Y^{2}, Z^{1}\right)$ and join it to vertices $z_{4 r-2}^{1}$ and $z_{4 r-1}^{1}$. Place $z_{4 p+1}^{1}$ in face 1 of $G_{r}\left(Y^{2}, Z^{1}\right)$ and join it to vertices $y_{4 r-2}^{2}$ and $y_{4 r-1}^{2}$.

Step 5: Add the vertices $z_{4 p+1}^{1}$ and $x_{4 p+1}^{2}$ to graph $G_{r}\left(Z^{1}, X^{2}\right)$.

Place $z_{4 p+1}^{1}$ in face 1 of $G_{r}\left(Z^{1}, X^{2}\right)$ and join it to vertices $x_{4 r-3}^{2}$ and $x_{4 r}^{2}$. Place $x_{4 p+1}^{2}$ in face 3 of $G_{r}\left(Z^{1}, X^{2}\right)$ and join it to vertices $z_{4 r-3}^{1}$ and $z_{4 r}^{1}$.

Step 6: Add the vertices $z_{4 p+1}^{2}$ and $x_{4 p+1}^{1}$ to graph $G_{r}\left(Z^{2}, X^{1}\right)$.

Place $z_{4 p+1}^{2}$ in face 1 of $G_{r}\left(Z^{2}, X^{1}\right)$ and join it to vertices $x_{4 r-3}^{1}$ and $x_{4 r}^{1}$. Place $x_{4 p+1}^{1}$ in face 3 of $G_{r}\left(Z^{2}, X^{1}\right)$ and join it to vertices $z_{4 r-3}^{2}$ and $z_{4 r}^{2}$.

We denote the above graphs we obtain from Steps $1-6$ by $\widehat{G}_{r}\left(X^{1}, Y^{2}\right), \widehat{G}_{r}\left(X^{2}, Y^{1}\right)$, $\widehat{G}_{r}\left(Y^{1}, Z^{2}\right), \widehat{G}_{r}\left(Y^{2}, Z^{1}\right), \widehat{G}_{r}\left(Z^{1}, X^{2}\right)$ and $\widehat{G}_{r}\left(Z^{2}, X^{1}\right)$ respectively.

Let

$$
\widehat{G}_{r}^{1}=\widehat{G}_{r}\left(X^{1}, Y^{2}\right) \cup \widehat{G}_{r}\left(Y^{1}, Z^{2}\right) \cup \widehat{G}_{r}\left(Z^{1}, X^{2}\right)
$$

and

$$
\widehat{G}_{r}^{2}=\widehat{G}_{r}\left(X^{2}, Y^{1}\right) \cup \widehat{G}_{r}\left(Y^{2}, Z^{1}\right) \cup \widehat{G}_{r}\left(Z^{2}, X^{1}\right) .
$$

Step 7: Add the edges $z_{4 r}^{1} x_{4 r}^{2}, y_{4 r-1}^{1} z_{4 r-1}^{2}, z_{4 r-2}^{1} y_{4 r-2}^{2}, x_{4 r-3}^{1} z_{4 r-3}^{2}$ and $z_{4 r}^{2} x_{4 r}^{1}$, $y_{4 r-1}^{2} z_{4 r-1}^{1}, z_{4 r-2}^{2} y_{4 r-2}^{1}, x_{4 r-3}^{2} z_{4 r-3}^{1}$ to graphs $\widehat{G}_{r}^{1}$ and $\widehat{G}_{r}^{2}$ respectively, $1 \leq r \leq p$.

For graph $\widehat{G}_{r}\left(Y^{1}, Z^{2}\right) \subset \widehat{G}_{r}^{1}$, we delete the edge $y_{4 r-3}^{1} z_{4 r}^{2}$ and join the vertex $y_{4 r-1}^{1}$ to vertex $z_{4 r-1}^{2}$, then we get a planar graph $\widetilde{G}_{r}\left(Y^{1}, Z^{2}\right)$. According to Lemma 4.3 , the graph $\widetilde{G}_{r}\left(Y^{1}, Z^{2}\right)$ has a planar embedding whose outer face has the same boundary as face 2, then the vertex $z_{4 r-3}^{2}$ is on the boundary of this outer face.

For graph $\widehat{G}_{r}\left(Z^{1}, X^{2}\right) \subset \widehat{G}_{r}^{1}$, delete the edge $z_{4 r-2}^{1} x_{4 r-1}^{2}$ and join $z_{4 r}^{1}$ to $x_{4 r}^{2}$, then we get a planar graph $\widetilde{G}_{r}\left(Z^{1}, X^{2}\right)$. According to Lemma 4.3, the graph $\widetilde{G}_{r}\left(Z^{1}, X^{2}\right)$ has a planar embedding whose outer face has boundary as $z_{4 r}^{1} x_{4 r}^{2} z_{4 r-2}^{1} x_{i}^{2} z_{4 r}^{1}\left(x_{i}^{2} \in \bigcup_{i=1, i \neq r}^{p}\left\{x_{4 i-1}^{2}\right.\right.$, $\left.x_{4 i}^{2}\right\}$ ), then the vertex $z_{4 r-2}^{1}$ is on the boundary of this outer face.

Since the vertices $x_{4 r-3}^{1}$ and $y_{4 r-2}^{2}$ are on the boundary of the outer face of the embedding of $\widehat{G}_{r}\left(X^{1}, Y^{2}\right) \subset \widehat{G}_{r}^{1}$, we can join $x_{4 r-3}^{1}$ to $z_{4 r-3}^{2}, y_{4 r-2}^{2}$ to $z_{4 r-2}^{1}$ without edge crossing. Then we get a planar graph $\widetilde{G}_{r}^{1}$.

With the same process, for the graph $G_{r}^{2}$, we delete edges $y_{4 r-3}^{2} z_{4 r}^{1}$ and $z_{4 r-2}^{2} x_{4 r-1}^{1}$, join $y_{4 r-1}^{2}$ to $z_{4 r-1}^{1}$, join $z_{4 r}^{2}$ to $x_{4 r}^{1}$, join $x_{4 r-3}^{2}$ to $z_{4 r-3}^{1}$ and join $y_{4 r-2}^{1}$ to $z_{4 r-2}^{2}$, then we get a planar graph $\widetilde{G}_{r}^{2}$.

Table 1 shows the edges that we add to $G_{r}^{1}$ and $G_{r}^{2}(1 \leq r \leq p)$ in Steps $1-7$.

Step 8: The remaining edges form a planar graph $\widetilde{G}_{p+1}$.

The edges that belong to $K_{4 p+1,4 p+1,4 p+1} \times K_{2}$ but not to any $\widetilde{G}_{r}^{1}, \widetilde{G}_{r}^{2}(1 \leq r \leq p)$ are shown in Table 2, in which the edges in the last two rows list the edges deleted in Step 7. The remaining edges form a graph, denote by $\widetilde{G}_{p+1}$. We draw a planar embedding of $\widetilde{G}_{p+1}$ in Figure 8, so $\widetilde{G}_{p+1}$ is a planar graph. 
Table 1: The edges we add to $G_{r}^{1}$ and $G_{r}^{2}(1 \leq r \leq p)$.

\begin{tabular}{lc|c}
\multicolumn{2}{c|}{ Edges } & Subscript \\
\hline \hline$x_{4 p+1}^{1} y_{i}^{2}, x_{4 p+1}^{2} y_{i}^{1}$, & $z_{4 p+1}^{1} x_{i}^{2}, z_{4 p+1}^{2} x_{i}^{1}$, & \\
$x_{4 p+1}^{1} z_{i}^{2}, x_{4 p+1}^{2} z_{i}^{1}$, & $x_{i}^{1} z_{i}^{2}, x_{i}^{2} z_{i}^{1}$, & $i=4 r-3,4 r$. \\
\hline$y_{4 p+1}^{1} z_{i}^{2}, y_{4 p+1}^{2} z_{i}^{1}$, & $z_{4 p+1}^{1} y_{i}^{2}, z_{4 p+1}^{2} y_{i}^{1}$, & \\
$y_{4 p+1}^{1} x_{i}^{2}, y_{4 p+1}^{2} x_{i}^{1}$, & $y_{i}^{1} z_{i}^{2}, y_{i}^{2} z_{i}^{1}$, & $i=4 r-2,4 r-1$.
\end{tabular}

Table 2: The edges of $\widetilde{G}_{p+1}$.

\begin{tabular}{c|c} 
Edges & Subscript $(1 \leq r \leq p)$ \\
\hline \hline$x_{4 p+1}^{1} y_{i}^{2}, x_{4 p+1}^{2} y_{i}^{1}, \quad z_{4 p+1}^{1} x_{i}^{2}, z_{4 p+1}^{2} x_{i}^{1}$, & \\
$x_{4 p+1}^{1} z_{i}^{2}, x_{4 p+1}^{2} z_{i}^{1}, \quad x_{i}^{1} z_{i}^{2}, x_{i}^{2} z_{i}^{1}$, & $i=4 r-2,4 r-1$. \\
\hline$y_{4 p+1}^{1} z_{i}^{2}, y_{4 p+1}^{2} z_{i}^{1}, \quad z_{4 p+1}^{1} y_{i}^{2}, z_{4 p+1}^{2} y_{i}^{1}$, & $i=4 r-3,4 r$. \\
$y_{4 p+1}^{1} x_{i}^{2}, y_{4 p+1}^{2} x_{i}^{1}, \quad y_{i}^{1} z_{i}^{2}, y_{i}^{2} z_{i}^{1}$, & $i=4 r-3,4 r-2,4 r-1,4 r$. \\
\hline$x_{i}^{1} y_{i}^{2}, x_{i}^{2} y_{i}^{1}$, & $i=4 p+1$. \\
\hline$x_{i}^{1} y_{i}^{2}, y_{i}^{2} z_{i}^{1}, z_{i}^{1} x_{i}^{2}, x_{i}^{2} y_{i}^{1}, y_{i}^{1} z_{i}^{2}, z_{i}^{2} x_{i}^{1}$, & $i=4 r-3, j=4 r$. \\
\hline$y_{i}^{1} z_{j}^{2}, y_{i}^{2} z_{j}^{1}$, & $i=4 r-2, j=4 r-1$.
\end{tabular}

Therefore $\left\{\widetilde{G}_{1}^{1}, \ldots, \widetilde{G}_{p}^{1}, \widetilde{G}_{1}^{2}, \ldots, \widetilde{G}_{p}^{2}, \widetilde{G}_{p+1}\right\}$ is a planar decomposition of $K_{4 p+1,4 p+1,4 p+1} \times K_{2}$, the Lemma follows.

Figure 9 illustrates a planar decomposition of $K_{9,9,9} \times K_{2}$ with five subgraphs.

A graph $G$ is said to be thickness $t$-minimal, if $\theta(G)=t$ and every proper subgraphs of it have a thickness less than $t$.

Lemma 4.5. When $n=4 p+3$, there exists a planar decomposition of Kronecker product graph $K_{4 p+3,4 p+3,4 p+3} \times K_{2}$ with $2 p+2$ subgraphs.

Proof. Case 1. When $p=0$.

As shown in Figure 10, we give a planar decomposition of $K_{3,3,3} \times K_{2}$ with 2 subgraphs.

Case 2. When $p \geq 1$.

The graph $K_{4 p+3,4 p+3}$ is a thickness $(p+2)$-minimal graph. Hobbs, Grossman [11] and Bouwer, Broere [6] proved it independently, by giving two different planar subgraphs decompositions $\left\{H_{1}, \ldots, H_{p+2}\right\}$ of $K_{4 p+3,4 p+3}$ in which $H_{p+2}$ contains only one edge. Suppose that the two vertex parts of $K_{n, n}$ is $\left\{v_{1}, \ldots, v_{n}\right\}$ and $\left\{u_{1}, \ldots, u_{n}\right\}$, the only one edge in the $H_{p+2}$ is $v_{a} u_{b}$ (the edge is $v_{1} u_{1}$ in [11] and $v_{4 p+3} u_{4 p-1}$ in [6]). For $1 \leq i \leq p+2$, $H_{i}$ is a bipartite graph, so we also denote it by $H_{i}(V, U)$.

Because $K_{n, n, n} \times K_{2}=G^{1} \cup G^{2}$ in which $G^{1}=G\left(X^{1}, Y^{2}\right) \cup G\left(Y^{1}, Z^{2}\right) \cup G\left(Z^{1}, X^{2}\right)$ and $G^{2}=G\left(X^{2}, Y^{1}\right) \cup G\left(Y^{2}, Z^{1}\right) \cup G\left(Z^{2}, X^{1}\right),\left|X^{i}\right|=\left|Y^{i}\right|=\left|Z^{i}\right|=n(i=1,2)$, all the graphs $G\left(X^{1}, Y^{2}\right), G\left(Y^{1}, Z^{2}\right), G\left(Z^{1}, X^{2}\right), G\left(X^{2}, Y^{1}\right), G\left(Y^{2}, Z^{1}\right)$ and $G\left(Z^{2}, X^{1}\right)$ are isomorphic to $K_{n, n}$. 


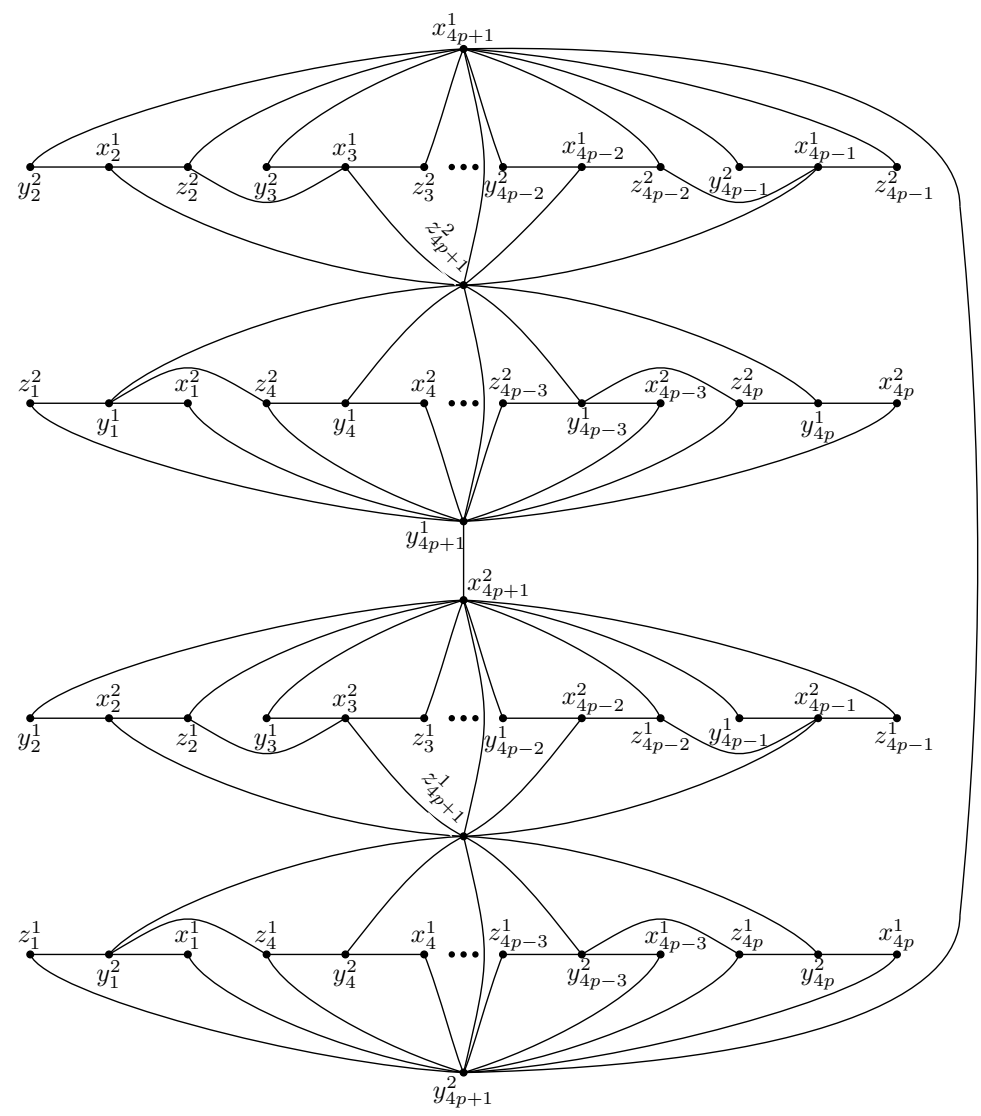

Figure 8: The graph $\widetilde{G}_{p+1}$.

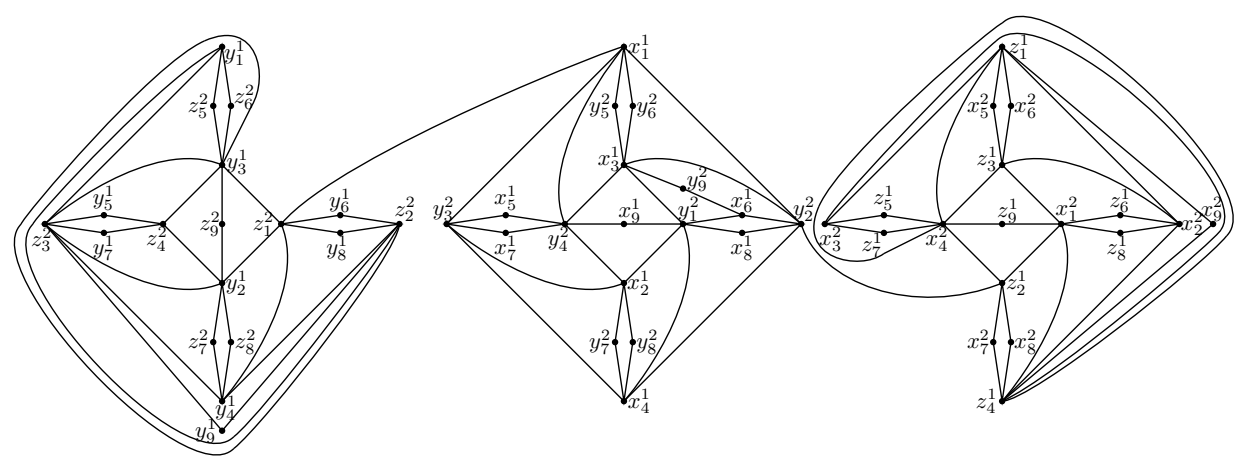

(a) The graph $\widetilde{G}_{1}^{1}$.

Figure 9: A planar decomposition of $K_{9,9,9} \times K_{2}$. 


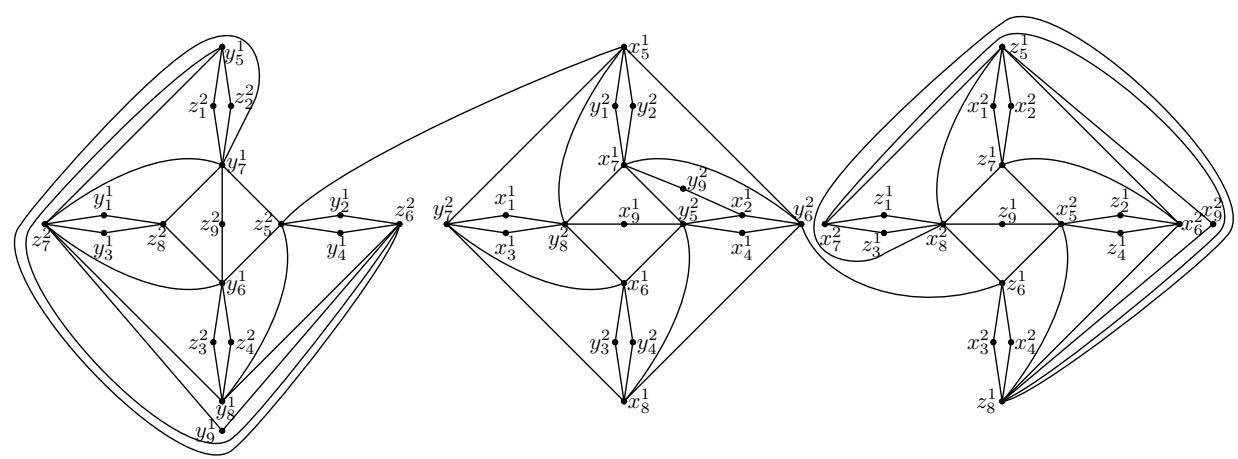

(b) The graph $\widetilde{G}_{2}^{1}$.

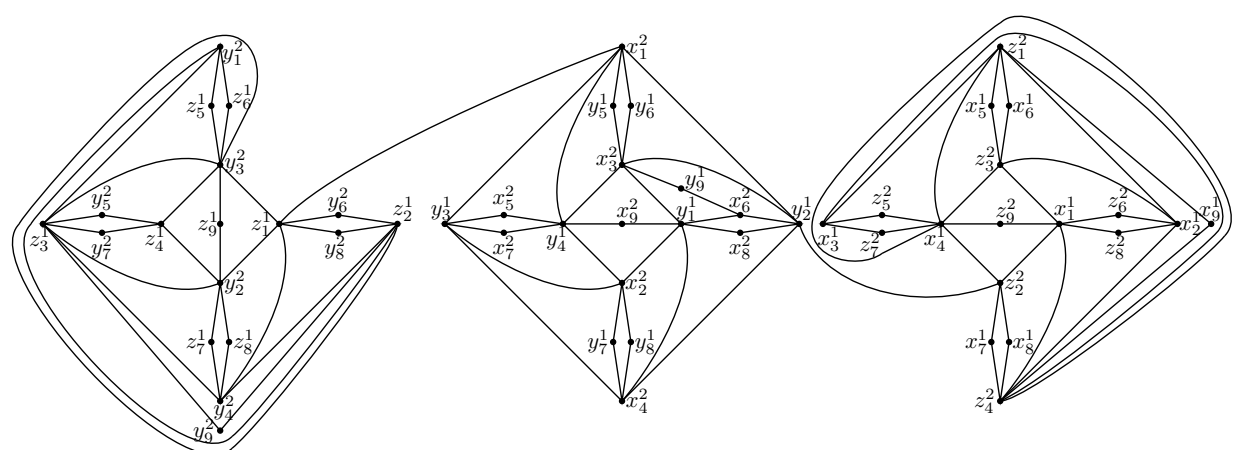

(c) The graph $\widetilde{G}_{1}^{2}$.

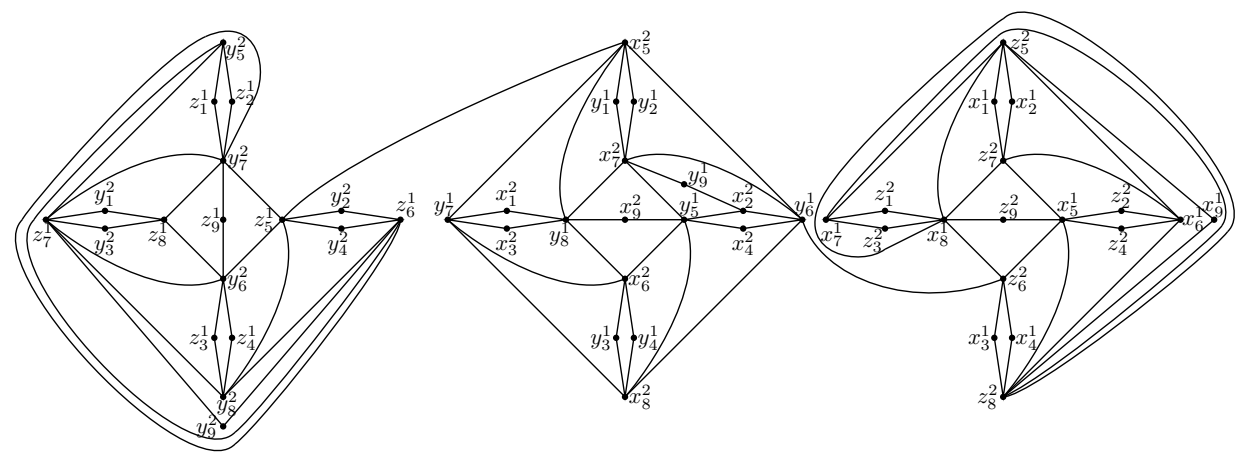

(d) The graph $\widetilde{G}_{2}^{2}$.

Figure 9: A planar decomposition of $K_{9,9,9} \times K_{2}$. 


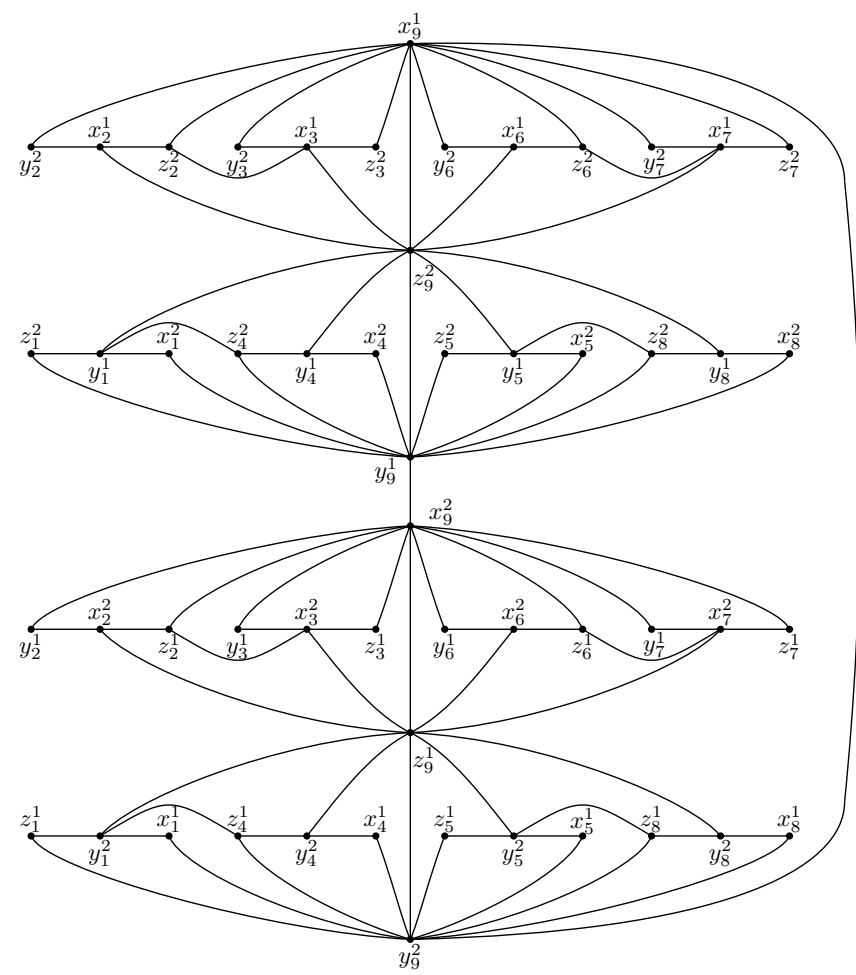

(e) The graph $\widetilde{G}_{3}$.

Figure 9: A planar decomposition of $K_{9,9,9} \times K_{2}$.
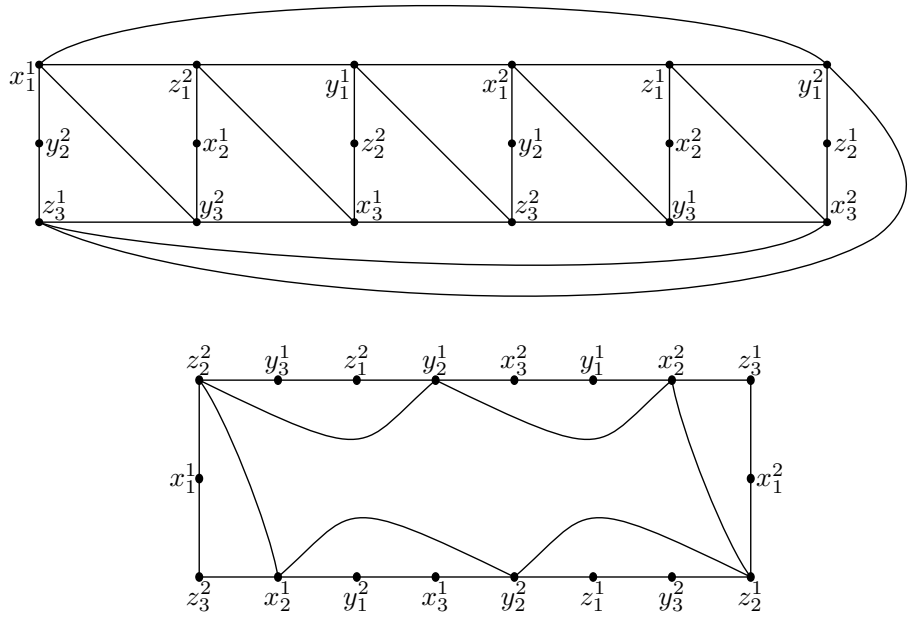

Figure 10: A planar decomposition of $K_{3,3,3} \times K_{2}$. 
For graph $H_{i}(V, U)(1 \leq i \leq p+2)$, we replace the vertex set $V$ by $X^{1}, U$ by $Y^{2}$, i.e., for each $1 \leq t \leq n$, replace the vertex $v_{t}$ by $x_{t}^{1}$, and $u_{t}$ by $y_{t}^{2}$, then we get a graph $H_{i}\left(X^{1}, Y^{2}\right)$. Analogously, we can obtain graphs $H_{i}\left(Y^{1}, Z^{2}\right), H_{i}\left(Z^{1}, X^{2}\right), H_{i}\left(X^{2}, Y^{1}\right)$, $H_{i}\left(Y^{2}, Z^{1}\right)$ and $H_{i}\left(Z^{2}, X^{1}\right)$. For $1 \leq i \leq p+2$, let

$$
H_{i}^{1}=H_{i}\left(X^{1}, Y^{2}\right) \cup H_{i}\left(Y^{1}, Z^{2}\right) \cup H_{i}\left(Z^{1}, X^{2}\right),
$$

then $H_{i}^{1}$ is a planar graph, because $H_{i}\left(X^{1}, Y^{2}\right), H_{i}\left(Y^{1}, Z^{2}\right), H_{i}\left(Z^{1}, X^{2}\right)$ are disjoint with each other. For the same reason, the graph

$$
H_{i}^{2}=H_{i}\left(X^{2}, Y^{1}\right) \cup H_{i}\left(Y^{2}, Z^{1}\right) \cup H_{i}\left(Z^{2}, X^{1}\right)
$$

is also a planar graph, $1 \leq i \leq p+2$. And we have

$$
K_{4 p+3,4 p+3,4 p+3} \times K_{2}=G^{1} \cup G^{2}=\bigcup_{i=1}^{p+2}\left(H_{i}^{1} \cup H_{i}^{2}\right),
$$

in which $E\left(H_{p+2}^{1}\right)=\left\{x_{a}^{1} y_{b}^{2}, y_{a}^{1} z_{b}^{2}, z_{a}^{1} x_{b}^{2}\right\}$ and $E\left(H_{p+2}^{2}\right)=\left\{x_{a}^{2} y_{b}^{1}, y_{a}^{2} z_{b}^{1}, z_{a}^{2} x_{b}^{1}\right\}$.

In the following, we will add edges in $E\left(H_{p+2}^{1}\right)$ to graphs $H_{1}^{2}$ and $H_{2}^{2}$, add edges in $E\left(H_{p+2}^{2}\right)$ to graphs $H_{1}^{1}$ and $H_{1}^{2}$ to complete the proof. From Lemma 4.3, there exists a planar embedding of $H_{1}\left(Y^{1}, Z^{2}\right)$ such that vertex $z_{a}^{2}$ on the boundary of its outer face, exists a planar embedding of $H_{1}\left(X^{1}, Y^{2}\right)$ such that $x_{b}^{1}$ on the boundary of its outer face. Then we join $z_{a}^{2}$ to $x_{b}^{1}$ without edge crossing. Suppose $y_{b}^{1}$ is on the boundary of inner face $F$ of the embedding of $H_{1}\left(Y^{1}, Z^{2}\right)$, put the embedding of $H_{1}\left(Z^{1}, X^{2}\right)$ in face $F$ with $x_{a}^{2}$ on the boundary of its outer face, then we join $x_{a}^{2}$ to $y_{b}^{1}$ without edge crossing. After adding both $x_{a}^{2} y_{b}^{1}$ and $z_{a}^{2} x_{b}^{1}$ to $H_{1}^{1}$ without edge crossing, we get a planar graph $\widetilde{H}_{1}^{1}$. With the same process, we add both $x_{a}^{1} y_{b}^{2}$ and $z_{a}^{1} z_{b}^{2}$ to $H_{1}^{2}$ without edge crossing, then we get a planar graph $\widetilde{H}_{1}^{2}$. From Lemma 4.3 , we can also add $y_{a}^{2} z_{b}^{1}$ to $H_{2}^{1}$, and $y_{a}^{1} z_{b}^{2}$ to $H_{2}^{2}$ without edge crossing, then we get planar graphs $\widetilde{H}_{2}^{1}$ and $\widetilde{H}_{2}^{2}$ respectively.

Then we get a planar decomposition

$$
\left\{\widetilde{H}_{1}^{1}, \widetilde{H}_{2}^{1}, H_{3}^{1}, \ldots, H_{p+1}^{1}, \widetilde{H}_{1}^{2}, \widetilde{H}_{2}^{2}, H_{3}^{2}, \ldots, H_{p+1}^{2}\right\}
$$

of $K_{4 p+3,4 p+3,4 p+3} \times K_{2}$ with $2 p+2$ subgraphs.

Summarizing Cases 1 and 2, the lemma follows.

Theorem 4.6. The thickness of the Kronecker product of $K_{n, n, n}$ and $K_{2}$ is

$$
\theta\left(K_{n, n, n} \times K_{2}\right)=\left\lceil\frac{n+1}{2}\right\rceil .
$$

Proof. Because of $E\left(K_{n, n, n} \times K_{2}\right)=6 n^{2}$ and $V\left(K_{n, n, n} \times K_{2}\right)=6 n$, from Theorem 2.2, we have

$$
\theta\left(K_{n, n, n} \times K_{2}\right) \geq\left\lceil\frac{6 n^{2}}{2(6 n)-4}\right\rceil=\left\lceil\frac{n}{2}+\frac{n}{6 n-2}\right\rceil=\left\lceil\frac{n+1}{2}\right\rceil .
$$

When $n=4 p+2$, because $K_{4 p+2,4 p+2,4 p+2} \times K_{2}$ is a subgraph of $K_{4 p+3,4 p+3,4 p+3} \times$ $K_{2}$, we have $\theta\left(K_{4 p+2,4 p+2,4 p+2} \times K_{2}\right) \leq \theta\left(K_{4 p+3,4 p+3,4 p+3} \times K_{2}\right)$. Combining this fact with Lemmas 4.2, 4.4 and 4.5, we have

$$
\theta\left(K_{n, n, n} \times K_{2}\right) \leq\left\lceil\frac{n+1}{2}\right\rceil .
$$

From inequalities (4.1) and (4.2), the theorem is obtained. 


\section{ORCID iDs}

Xia Guo (D) https://orcid.org/0000-0003-0217-105X

Yan Yang (D) https://orcid.org/0000-0002-9666-5167

\section{References}

[1] V. B. Alekseev and V. S. Gončakov, The thickness of an arbitrary complete graph, Mat. Sb. (N.S.) 101(143) (1976), 212-230, doi:10.1070/sm1976v030n02abeh002267.

[2] L. Beaudou, P. Dorbec, S. Gravier and P. K. Jha, On planarity of direct product of multipartite complete graphs, Discrete Math. Algorithms Appl. 1 (2009), 85-104, doi:10.1142/ s179383090900004x.

[3] L. W. Beineke and F. Harary, The thickness of the complete graph, Canadian J. Math. 17 (1965), 850-859, doi:10.4153/cjm-1965-084-2.

[4] L. W. Beineke, F. Harary and J. W. Moon, On the thickness of the complete bipartite graph, Proc. Cambridge Philos. Soc. 60 (1964), 1-5, doi:10.1017/s0305004100037385.

[5] J. A. Bondy and U. S. R. Murty, Graph Theory, volume 244 of Graduate Texts in Mathematics, Springer, New York, 2008, doi:10.1007/978-1-84628-970-5.

[6] I. Z. Bouwer and I. Broere, Note on $t$-minimal complete bipartite graphs, Canad. Math. Bull. 11 (1968), 729-732, doi:10.4153/cmb-1968-088-x.

[7] Y. Chen and Y. Yang, The thickness of the complete multipartite graphs and the join of graphs, J. Comb. Optim. 34 (2017), 194-202, doi:10.1007/s10878-016-0057-1.

[8] Y. Chen and X. Yin, The thickness of the Cartesian product of two graphs, Canad. Math. Bull. 59 (2016), 705-720, doi:10.4153/cmb-2016-020-1.

[9] D. Duffus, B. Sands and R. E. Woodrow, On the chromatic number of the product of graphs, $J$. Graph Theory 9 (1985), 487-495, doi:10.1002/jgt.3190090409.

[10] M. Farzan and D. A. Waller, Kronecker products and local joins of graphs, Canadian J. Math. 29 (1977), 255-269, doi:10.4153/cjm-1977-027-1.

[11] A. M. Hobbs and J. W. Grossman, A class of thickness-minimal graphs, J. Res. Nat. Bur. Standards Sect. B 72B (1968), 145-153, https://nvlpubs.nist.gov/nistpubs/ jres/72B/jresv72Bn2p145_A1b.pdf.

[12] S. Klavžar, Coloring graph products—a survey, Discrete Math. 155 (1996), 135-145, doi:10. 1016/0012-365x(94)00377-u.

[13] M. Kleinert, Die Dicke des n-dimensionalen Würfel-Graphen, J. Comb. Theory 3 (1967), 1015, doi:10.1016/s0021-9800(67)80010-3.

[14] J. Leskovec, D. Chakrabarti, J. Kleinberg, C. Faloutsos and Z. Ghahramani, Kronecker graphs: an approach to modeling networks, J. Mach. Learn. Res. 11 (2010), 985-1042, https: // www.jmlr.org/papers/vil/leskovec10a.html.

[15] P. Mutzel, T. Odenthal and M. Scharbrodt, The thickness of graphs: a survey, Graphs Combin. 14 (1998), 59-73, doi:10.1007/p100007219.

[16] W. T. Tutte, The thickness of a graph, Indag. Math. 66 (1963), 567-577, doi:10.1016/ s1385-7258(63)50055-9.

[17] J. M. Vasak, The Thickness of the Complete Graph, Ph.D. thesis, University of Illinois at Urbana-Champaign, 1976, https: / / www . proquest. com/docview/302820090.

[18] W. Wang and Z. Yan, Connectivity of Kronecker products with complete multipartite graphs, Discrete Appl. Math. 161 (2013), 1655-1659, doi:10.1016/j.dam.2013.01.009. 
[19] Y. Yang, Remarks on the thickness of $K_{n, n, n}$, Ars Math. Contemp. 12 (2017), 135-144, doi: 10.26493/1855-3974.823.068.

[20] Y. Yang and Y. Chen, The thickness of amalgamations and Cartesian product of graphs, Discuss. Math. Graph Theory 37 (2017), 561-572, doi:10.7151/dmgt.1942. 